Radioactive Threat Detection with Scattering Physics: A Model-Based Application

J. V. Candy, D. H. Chambers, E. F. Breitfeller, B. L. Guidry, J. M. Verbeke, M. A. Axelrod, K. E. Sale, A. M. Meyer

February 5, 2010

IAPR/IEEE Conference on Cognitive Information Proceesing Elba Island, Italy June 14, 2010 through June 16, 2010 
This document was prepared as an account of work sponsored by an agency of the United States government. Neither the United States government nor Lawrence Livermore National Security, LLC, nor any of their employees makes any warranty, expressed or implied, or assumes any legal liability or responsibility for the accuracy, completeness, or usefulness of any information, apparatus, product, or process disclosed, or represents that its use would not infringe privately owned rights. Reference herein to any specific commercial product, process, or service by trade name, trademark, manufacturer, or otherwise does not necessarily constitute or imply its endorsement, recommendation, or favoring by the United States government or Lawrence Livermore National Security, LLC. The views and opinions of authors expressed herein do not necessarily state or reflect those of the United States government or Lawrence Livermore National Security, LLC, and shall not be used for advertising or product endorsement purposes. 


\title{
Radioactive Threat Detection with Scattering Physics: a Model-Based Application
}

\author{
J. V. Candy, Fellow, IEEE, D. H. Chambers, Senior Member, IEEE, E. F. Breitfeller, Member, IEEE, B. L. Guidry, \\ Member, IEEE, J. M. Verbeke, M. A. Axelrod, K. E. Sale and A. M. Meyer Senior Member, IEEE
}

\begin{abstract}
The detection of radioactive contraband is a critical problem in maintaining national security for any country. Emissions from threat materials challenge both detection and measurement technologies especially when concealed by various types of shielding complicating the transport physics significantly. The development of a model-based sequential Bayesian processor that captures both the underlying transport physics including scattering offers a physics-based approach to attack this challenging problem. It is shown that this processor can be used to develop an effective detection technique.
\end{abstract}

\section{INTRODUCTION}

A commercial semi-trailer truck passing through a portal monitor at a reasonable speed must be screened at ports-ofentry for possible threats emanating from radioactive contraband. Similarly, a container moving on a conveyor belt for relocation and screening at airports or shipping ports presents a potential mechanism to transport terrorist contraband into a country posing a significant threat to national security. The need to investigate techniques that can provide for more sensitive detection of terrorist threats throughout the world demand that meaningful approaches be developed to solving many critical security problems for the protection of valuable national resources.

Detection of threat radiological materials is a difficult problem primarily because of low observable count rates and short detection intervals available. For instance, semi-trailer vehicles move through portal systems allowing less than 10 seconds for the initial screening. Shielding materials from packaging and adjacent cargo present major difficulties in these low-count, hostile environments. Low-count detection is a challenging problem made difficult because of background noise, measurement system inadequacies, and the heterogeneous transport paths between source and detector [1]-[3]. The basic problem we investigate is the detection and identification of radioactive contraband from low-count measurements using a physicsbased statistical approach based on Bayesian inference and model-based signal processing [4], [5]. Some work has been accomplished on this problem [6]-[8], but very little effort has been performed on this specific application in the signal processing area. Our approach closely follows the development of the Bayesian processor of a previous paper [8] with the incorporation of Compton scattering photons into the processing scheme. This is accomplished by extending the radionuclide representation to incorporate the scattered photons along with the development of a simplified one-dimensional transport model required to capture material interactions in the shield and detector materials.

In Sec. II we develop the physics-based signal processing models employed in the subsequent Bayesian constructs. Next we develop the sequential detection paradigm in Sec. III. In Sec. IV we investigate solutions to the processing problem and implementation of the technique. In Sec. V we develop the overall identification scheme and demonstrate its performance on experimental data. The results of applying the processor to controlled experimental data shows the capability of the sequential processor to perform in an effective manner.

\section{PHYSICS-BASED PROCESSING MODEL}

In this section we discuss physics-based representations that will be incorporated into the model-based signal processors for detection and identification. The measured data consists of a low energy count, random, impulsive-like, time series measurements (energy vs time) in the form of an event mode sequence (EMS) obtained from the detector electronics [2]. A particular radionuclide can be uniquely characterized by two basic parameters: its energy and the relative intensity of $\gamma$-rays emitted [2]. Mathematically, we define the pair, $\left[\left\{\epsilon_{m \ell}\right\},\left\{\lambda_{m \ell}\right\}\right]$, as the respective energy $(\mathrm{MeV})$ and photon detection rate of the $\ell$ th-downscatter and $m$ th $\gamma$-ray line of the radionuclide. The $\gamma$-ray is transported through a medium and interacts with materials, shield and detector resulting in the output of a list of events consisting of an event time an amplitude of pulse height or energy.

For sequential detection we process each event in the EMS individually using a model with parameters that are analogous to the features in a pulse-height spectrum $(P H S)$ of counts versus energy. Since a radionuclide emits $\gamma$-rays at specific energies and rates, the sequence of emitted photons can be characterized by sets of measured energies and arrival times at the detector: $\left[\left\{\epsilon_{m \ell}\right\},\left\{\tau_{m \ell}\right\}\right], \quad \ell=0, \cdots, L_{\epsilon}(m), m=$ $1, \cdots, M_{\epsilon}$. The index $m$ represents the $m$ th $\gamma$-ray line (energy) for a radionuclide with $M_{\epsilon}$ lines and the index $\ell>1$ represents the corresponding $\ell$ th "downscattered" line. It is convenient to think of each line as a separate source $(\ell=0)$. Then the emission from a given radionuclide is represented as a superposition of individual $\gamma$-ray sources. We will refer to this as the monoenergetic decomposition of the EMS for the radionuclide. 


\section{A. Event Mode Sequence}

We start with a single $\gamma$-ray arrival which can (in our problem) be either an absorbed photon that exchanges all of its energy with an electron (photoelectric absorption) or a scattered photon that exchanges part of its energy with an electron (Compton scattering). In either case, we define $\xi\left(n ; \epsilon_{m \ell}, \tau_{m \ell}\right)$, a component of an EMS sequence, as the $n$th measured photon arrival from the $m$ th monoenergetic source of energy $(\ell=0)$ or the $\ell$ th downscatter $(\ell>1)$ of energy $\epsilon_{m \ell}(n)$, at arrival time, $\tau_{m \ell}(n)$ with associated detection rate, $\lambda_{m \ell}(n)$. The finite resolution of the detector introduces a random component to the measured energy; therefore, the energy is more accurately represented as a random variable at the $n$th arrival $\epsilon_{m \ell}(n)$. The resulting representation for a single photon arrival is $\xi\left(n ; \epsilon_{m \ell}, \tau_{m \ell}\right)=\epsilon_{m \ell}(n) \delta\left(t-\tau_{m \ell}(n)\right)$. By photon arrival we refer only to the arrival of photons that are measured by the detector and contribute to the EMS output of the detector.

In order to define the entire emission sequence over a specified observation time interval, $\left[t_{o}, T\right)$, we introduce the set notation, $\tilde{\tau}_{m \ell}:=\left\{\tau_{m \ell}(1) \cdots \tau_{m \ell}\left(N_{\epsilon}(m)\right)\right\}$ and $\tilde{\epsilon}_{m \ell}:=$ $\left\{\epsilon_{m \ell}(1) \cdots \epsilon_{m \ell}\left(N_{\epsilon}(m)\right)\right\}$ for $; \ell=0,1, \cdots, L_{\epsilon}(m) ; m=$ $0, \cdots, M_{\epsilon}$ with $N_{\epsilon}(m)$ along with $L_{\epsilon}(m)$ as the total number of counts and downscatterers for the $m$ th-source in the interval. Therefore, $\xi\left(n ; \tilde{\epsilon}_{m \ell}, \tilde{\tau}_{m \ell}\right)$ results in an impulse train of random energies from the $m \ell$ th source up to the $n$th arrival.

The interarrival time is defined by $\triangle \tau_{m \ell}(n)=\tau_{m \ell}(n)-$ $\tau_{m \ell}(n-1)$ for $\triangle \tau_{m \ell}(0)=t_{o}$ with the corresponding set definition (above) of $\triangle \tilde{\tau}_{m \ell}(n)$. Using this definition, we can rewrite with a slight abuse of notation, the photon arrival as $\xi\left(n ; \epsilon_{m \ell}, \tau_{m \ell}\right) \Rightarrow \xi\left(n ; \epsilon_{m \ell}, \triangle \tau_{m \ell}\right)=\epsilon_{m \ell}(n) \delta\left(t-\tau_{m \ell}(n)\right)$, where it is understood that $\tau_{m \ell}(n)=\tau_{m \ell}(n-1)+\triangle \tau_{m \ell}(n)$. Thus, we can rewrite the EMS in terms of interarrivals just as easily as arrivals, that is, the $m$ th monoenergetic source representation of a radionuclide characterized by its unique set of energy/arrival pairs is given by

$$
\xi\left(N_{\epsilon}(m) ; \tilde{\epsilon}_{m \ell}, \triangle \tilde{\tau}_{m \ell}\right)=\sum_{\ell=0}^{L_{\epsilon}(m)} \sum_{n=1}^{N_{\epsilon}(m)} \epsilon_{m \ell}(n) \delta\left(t-\triangle \tau_{m \ell}(n)\right)
$$

at detection rate $\lambda_{m \ell}(n)$ for $t_{o}$ known.

Following Refr. [8] this EMS model can be extended from a single monoenergetic source representation to incorporate a set of $M_{\epsilon}$-monoenergetic source components that compose a complete source radionuclide $(R N)$. The "extended" monoenergetic decomposition includes both photoelectric and downscattered photons. The emission of photons follows a well-defined probability structure, that is, since only one photon is emitted for each event there is a fixed probability (absolute intensity $\alpha_{m \ell}$ ) that the photon is emitted with energy $\epsilon_{m \ell}$ out of $L_{\epsilon} M_{\epsilon}$ possibilities. The probabilities for a given radionuclide are specified in its energy decay diagram [2], [3]. Therefore, we model this decay structure by incorporating an indicator function defined by [10]:

$$
\mathcal{I}_{j k}(m, \ell)=\left\{\begin{array}{cc}
1 & m=j \& \ell=k \\
0 & m \neq j \oplus \ell \neq k
\end{array}\right.
$$

where $\mathcal{I}_{j k}(m, \ell)$ is a random variable such that $\operatorname{Pr}\left(\mathcal{I}_{j k}(m, \ell)=\right.$ $1 \mid \xi(n ; \underline{\epsilon}, \underline{\tau}))=\operatorname{Pr}\left(\mathcal{I}_{j k}(m, \ell)=1 \mid \Xi_{n}\right)=\alpha_{j k}$ for $\alpha_{j k}$ the corresponding absolute intensity emission/occurrence probability of the $j$ th-monoenergetic radionuclide component and the $k$ th downscatter. Using the indicator function we can write the $j k$ th arrival $(m \rightarrow j ; \ell \rightarrow k$ ) of the EMS as

$$
\begin{aligned}
& \mathcal{R}(N ; \underline{\epsilon}, \Delta \underline{\tau})= \\
& \sum_{m=1}^{M_{\epsilon}} \sum_{\ell=0}^{L_{\epsilon}(m)} \sum_{n=1}^{N_{\epsilon}(m)} \mathcal{I}_{j k}(m, \ell) \epsilon_{m \ell}(n) \delta\left(t-\tau_{m \ell}(n)\right)
\end{aligned}
$$

for $\underline{\epsilon}:=\left\{\tilde{\epsilon}_{1 \ell}, \cdots, \tilde{\epsilon}_{M_{\epsilon} \ell}\right\}$, the complete set of energies composing $\mathcal{R}$ along with $\triangle \underline{\tau}:=\left\{\triangle \tilde{\tau}_{1 \ell}, \cdots, \tilde{\tau}_{M_{\epsilon} \ell}\right\}$, the corresponding set of interarrival times. The arrival index $N$ is the least upper bound of the set $N_{\epsilon}(m)$. We model the energy variations as Gaussian, $\epsilon \sim \mathcal{N}\left(\bar{\epsilon}_{m \ell}, \sigma_{\epsilon_{m \ell}}^{2}\right)$, the interarrivals as exponential, $\triangle \tau_{m \ell} \sim \mathcal{E}\left(\alpha_{m \ell} \lambda_{\triangle \tau_{m \ell}} \triangle \tau_{m \ell}(n)\right)$ [2] with the emission/occurrence probability as $\alpha_{m \ell}$.

\section{B. Compton Scattering Processing Model}

In this section we briefly discuss the simple $\gamma$-ray transport model to capture and discriminate downscattered photons. The fundamental idea is to represent the EMS as a marked Poisson process [6] specified by its rate parameter $\lambda(\epsilon)$ as a function of energy. Here the objective is to relate the source rate through the transport chain to the rate measured at the radiation detector.

The simplified $\gamma$-ray transport model developed for the model-based sequential processor is based on a simple onedimensional geometry describing the source radionuclide, shield effects and radiation detector including its material response function. The model incorporates the physics of photoelectric absorption and Compton scattering represented by the rate distributions. The general approach is to characterize the transport physics that incorporates the probabilities that the photon: (1) will escape from the material; (2) will downscatter to a lower energy; or (3) will be absorbed producing a photoelectron. Here the probability is a characteristic of both geometry and material composition. The resulting signal processing transport (SPT) model describes a fixed, onedimensional geometry of the source-shield-detector transport path assuming uncorrelated photon interaction ignoring the pair production physics prevalent at higher energy $(\epsilon>$ $1 \mathrm{MeV}$ ) [2].

The basis of this approach is the mathematical projection of the 6-dimensional Boltzmann radiation transport equation to a single dimensional point-to-point equivalent for a specific geometry incorporating shielding and the detector material response. Details of the model development can be found in Chambers [7]. 
The SPT model is represented by the $R N$ source, shield and detector with the output rate probability distributions defined by $\lambda_{S R C}(\epsilon), \lambda_{E S C}(\epsilon), \lambda_{D E T}(\epsilon)$, respectively. The source photon emission rate distribution corresponds to the distribution of photons incident (input) to either the shield or detector, $\lambda_{I N C}(\epsilon)$, depending on the transport configuration. A fraction or probability of the incident photons will be absorbed, $p_{A B S}(\epsilon)$ with corresponding rate distribution, $\lambda_{A B S}(\epsilon)$ given by: $\lambda_{A B S}(\epsilon)=p_{A B S}(\epsilon) \times \lambda_{I N C}(\epsilon)$ while the remaining photons escape or are scattered according to $\left(1-p_{A B S}\right) \times \lambda_{I N C}(\epsilon)$. Defining the escape probability as $p_{E S C}(\epsilon)$ enables us to write the corresponding scattering rate distribution as $\lambda_{E S C}(\epsilon)$.

To be more precise let $p_{S C A T}\left(\epsilon ; \epsilon^{\prime}\right)$ be the probability of photons at energy $\epsilon^{\prime}$ downscattered to $\epsilon$ by a single interaction with the material. The corresponding Compton scattered rate distribution can be expressed as

$\lambda_{S C A T}^{(1)}(\epsilon)=\mathcal{K}\left(\epsilon ; \epsilon^{\prime}\right) \circ \lambda_{I N C}\left(\epsilon^{\prime}\right)=\int_{\epsilon}^{\infty} \mathcal{K}\left(\epsilon ; \epsilon^{\prime}\right) \lambda_{I N C}\left(\epsilon^{\prime}\right) d \epsilon^{\prime}$

where $\mathcal{K}\left(\epsilon ; \epsilon^{\prime}\right):=p_{S C A T}\left(\epsilon ; \epsilon^{\prime}\right) \times\left(1-p_{A B S}\left(\epsilon^{\prime}\right)\right) \times(1-$ $\left.p_{E S C}\left(\epsilon^{\prime}\right)\right)$.

The total rate distributions of escaped and absorbed photons are obtained by summing over all $k$-scattering orders. That is, define the total scattering function as:

$$
\begin{aligned}
\lambda_{A B S}(\epsilon)= & p_{A B S}(\epsilon) \times \mathcal{S}_{S C A T}(\epsilon) \\
\lambda_{E S C}(\epsilon)= & p_{E S C}(\epsilon) \times\left(1-p_{A B S}(\epsilon)\right) \mathcal{S}_{S C A T}(\epsilon) \\
\lambda_{S C A T}(\epsilon)= & \int_{\epsilon}^{\infty} p_{S C A T}\left(\epsilon ; \epsilon^{\prime}\right) \times\left(1-p_{A B S}\left(\epsilon^{\prime}\right)\right) \\
& \times\left(1-p_{E S C}\left(\epsilon^{\prime}\right)\right) \mathcal{S}_{S C A T}\left(\epsilon^{\prime}\right) d \epsilon^{\prime}
\end{aligned}
$$

Solving these equations enable us to estimate the rate distributions of both absorbed and escaped photons for all orders of scattering. The model has been developed and validated using sophisticated Monte Carlo simulation algorithms (see [7] for more details).

From the signal processing perspective, we know that the sequential decision function (to follow) requires that for each photon arrival the energy, detection rate (reciprocal mean interarrival time) and emission/occurrence (photoelectric/downscatter) probabilities must be estimated. Since both photoelectric and downscattered arrivals can have the same energy but can have different monoenergetic target sources, it is the "scattering (occurrence) probability" or equivalently the emission probability (for photoelectrons) that must be estimated. These parameters act as inherent weighting functions and therefore each arrival can be thought of as being partitioned according to the various weights into the composite $R N$ decision function to make the decision.

\section{RADIONUCLIDE DETECTION}

The development of a radionuclide detector based on photon-by-photon processing follows. Thus, we develop a "sequential" technique that is aimed at processing a single photon arrival at a time rather than attempting to perform a

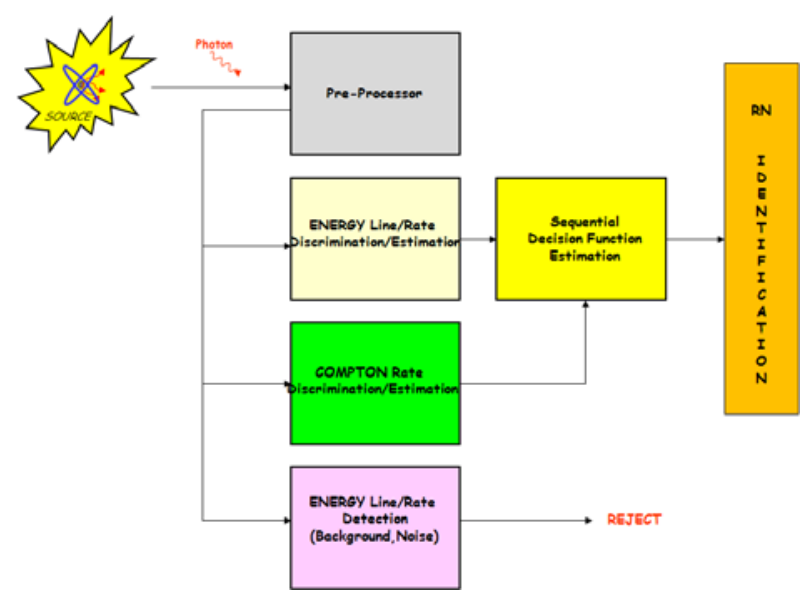

Fig. 1. Bayesian radiation detection: Acquisition, pre-processing (optional), energy/rate discrimination/estimation, Compton rate discrimination/estimation, background and extraneous line rejection, decision function estimation and $R N$ identification.

batch solution which is the basis of PHS analysis [2]. The basic approach is shown in Fig. 1. After a single photon is preprocessed by the measurement system, both energy and arrival time are extracted and passed onto energy/rate discriminators to determine whether or not it is a targeted photon or its downscatter. If acceptable (target), parameter estimates are sequentially updated and provided as input to the decision function for detection and identification. If not acceptable, the photon is rejected and discarded (noise or background).

To formally pose the radionuclide detection problem, we appeal to classical (sequential) detection theory [11]. We are to test the binary hypothesis that the measured EMS has evolved from the targeted radionuclide characterized uniquely from its monoenergetic decomposition of Eq. 2. Therefore, we specify the hypothesis test

$$
\begin{aligned}
& \mathcal{H}_{0}: \quad \xi(n ; \underline{\epsilon}, \triangle \underline{\tau})=\mathcal{R}(n ; \underline{\epsilon}, \triangle \underline{\tau})+\nu(n) \quad \text { [NON-TARGET] } \\
& \mathcal{H}_{1}: \quad \xi(n ; \underline{\epsilon}, \triangle \underline{\tau})=\mathcal{R}\left(n ; \underline{\epsilon}^{t}, \triangle \underline{\tau}^{t}\right)+\nu(n) \quad[\text { TARGET] }
\end{aligned}
$$

where $\mathcal{R}(n ; \underline{\epsilon}, \triangle \underline{\tau})$ is the random composite EMS of Eq. 2 contaminated with zero-mean, Gaussian measurement (instrumentation) noise, $\nu \sim \mathcal{N}\left(0, \sigma_{\nu}^{2}\right)$ for $\epsilon_{m \ell} \sim \mathcal{N}\left(\bar{\epsilon}_{m \ell}, \sigma_{\epsilon_{m \ell}}^{2}\right)$ and $\triangle \tau_{m \ell} \sim \mathcal{E}\left(\lambda_{\triangle \tau_{m \ell}} \Delta \tau_{m \ell}(n)\right)$. Here the superscript ${ }^{t}$ is used to denote the "true" or "target" parameters.

The optimal solution to this binary decision problem is based on applying the Neyman-Pearson theorem leading to the likelihood given by the ratio of probabilities [11]. However, since the distributions under investigation are members of the exponential family [10], then taking logarithms leads to the sequential log-likelihood ratio

$$
\begin{aligned}
\Lambda\left[\Xi_{n}\right] & =\Lambda\left[\Xi_{n-1}\right]+\ln \operatorname{Pr}\left(\xi(n ; \underline{\epsilon}, \triangle \underline{\tau}) \mid \Xi_{n-1}, \mathcal{H}_{1}\right) \\
& -\ln \operatorname{Pr}\left(\xi(n ; \underline{\epsilon}, \triangle \underline{\tau}) \mid \Xi_{n-1}, \mathcal{H}_{0}\right)
\end{aligned}
$$

and therefore, the Wald sequential probability-ratio test be- 
comes [11] (see Fig. 1)

$$
\begin{array}{clrl}
\Lambda\left[\Xi_{n}\right] & \geq \ln \mathcal{T}_{1}(n) & \text { Accept } \mathcal{H}_{1} \\
\ln \mathcal{T}_{0}(n) \leq \Lambda\left[\Xi_{n}\right] & \leq \ln \mathcal{T}_{1}(n) & & \text { Continue } \\
\Lambda\left[\Xi_{n}\right] & \leq \ln \mathcal{T}_{0}(n) & \text { Accept } \mathcal{H}_{0}
\end{array}
$$

where the set of EMS measurements are $\Xi_{N}$ := $\{\xi(0), \xi(1), \cdots, \xi(N)\}$ and the thresholds are specified in terms of the false alarm $\left(\mathrm{P}_{F A}\right)$ and miss $\left(\mathrm{P}_{M}\right)$ probabilities as

$$
\mathcal{T}_{0}(n)=\frac{\mathrm{P}_{M}(n)}{\mathrm{P}_{F A}(n)} \quad \mathcal{T}_{1}(n)=\frac{1-\mathrm{P}_{M}(n)}{\mathrm{P}_{F A}(n)}
$$

typically obtained by generating receiver operating characteristic $(R O C)$ curves and selecting an operating point $\left(P_{F A}, P_{M}\right)$. So we see that at each photon arrival (at time $n$ ), we can sequentially update the likelihood and thresholds to perform the detection - "photon-by-photon."

To implement this detection technique (see Eq. 5), we must specify the required distributions in order to calculate the decision function It can be shown that [8] the sequential loglikelihood ratio detector is given by

$$
\begin{aligned}
& \Lambda\left[\Xi_{n}\right]=\Lambda\left[\Xi_{n-1}\right]+ \\
& \sum_{m=1}^{M_{\epsilon}} \sum_{\ell=0}^{L_{\epsilon}(m)} \ln \Theta_{m \ell}\left(n ; \theta, \mathcal{H}_{1}\right)-\sum_{m=1}^{M_{\epsilon}} \sum_{\ell=0}^{L_{\epsilon}(m)} \ln \Theta_{m \ell}\left(n ; \theta, \mathcal{H}_{0}\right)
\end{aligned}
$$

where

$$
\begin{aligned}
& \Theta_{m \ell}\left(n ; \theta, \mathcal{H}_{i}\right):= \\
& \alpha_{m \ell} \times \operatorname{Pr}\left(\triangle \tau_{m \ell}(n) \mid \epsilon_{m \ell}(n), \mathcal{I}_{j k}(m, \ell), \Xi_{n-1}, \mathcal{H}_{i}\right) \times \\
& \operatorname{Pr}\left(\epsilon_{m \ell}(n) \mid \mathcal{I}_{j k}(m, \ell), \Xi_{n-1}, \mathcal{H}_{i}\right)
\end{aligned}
$$

and recall the emission/occurrence probability is

$$
\alpha_{m \ell}=\operatorname{Pr}\left(\mathcal{I}_{j k}(m, \ell) \mid \Xi_{n-1}, \mathcal{H}_{i}\right) ; \quad i=0,1
$$

giving us the general form required for our problem. Note that this formulation provides us with a channel-by-channel (photon-by-photon) processor, since the $m \ell$-th terms are available at the output of each channel.

It can also be shown [9] that by defining the photoelectric (absorption) log-likelihood as $\Lambda_{p e}\left[\Xi_{n-1}\right]$ and the downscatter log-likelihood as $\Lambda_{d s}\left[\Xi_{n-1}\right]$, we obtain the decomposition leading to the algorithmic structure (see Fig. 2)

$$
\Lambda\left[\Xi_{n}\right]=\Lambda\left[\Xi_{n-1}\right]+\Lambda_{p e}\left[\Xi_{n-1}\right]+\Lambda_{d s}\left[\Xi_{n-1}\right]
$$

where

$$
\begin{aligned}
\Lambda_{p e}\left[\Xi_{n-1}\right]:= & \sum_{m=1}^{M_{\epsilon}} \ln \Theta_{m 0}\left(n ; \theta, \mathcal{H}_{1}\right)-\ln \Theta_{m 0}\left(n ; \theta, \mathcal{H}_{0}\right) \\
\Lambda_{d s}\left[\Xi_{n-1}\right]:= & \sum_{m=1}^{M_{\epsilon}} \sum_{\ell=1}^{L_{\epsilon}(m)} \ln \Theta_{m \ell}\left(n ; \theta, \mathcal{H}_{1}\right) \\
& -\ln \Theta_{m \ell}\left(n ; \theta, \mathcal{H}_{0}\right)
\end{aligned}
$$

Not surprisingly, we see that this log-likelihood decomposition shows that if we ignore the downscattered photons as in Refr. [8] we cannot achieve the optimal decision because we are not using all of the physics information available! Thus, we expect the performance of this "extended" processor to be superior to the photoelectric only implementation!

The sequential radiation detection processor is illustrated in Fig. 2. As the photon arrives, its energy and interarrival time are extracted and discriminated to select the appropriate channel for processing. If it is a targeted photoelectron, then it is processed precisely as discussed in Refr. [8], that is, after discrimination the energy, interarrival (detection rate) and emission/occurrence probability parameters are estimated, the appropriate decision function updated and compared to the threshold to "decide" if the targeted $R N$ is present. On the other hand, if the arrival is not deemed a photoelectron, then it is discriminated to investigate the possibility of it being a potential downscattered arrival. Here discrimination is based on the selected energies associated with the target radionuclide and its corresponding Compton region. That is, for example with knowledge of the target $R N$ Compton edge [2], a number $\left(L_{\epsilon}(m)\right)$ of downscattered energies or bins are selected and used to specify a set of discriminants for Compton processing. If accepted, then parameter estimation is performed to enhance the energy which is used in the SPT model to provide an estimate of the corresponding detection rate and emission/occurrence probability. Thus, all of the downscatter parameters are updated along with the corresponding "true" decision functions. It is interesting to note that we can conceive of the downscattered photon information being partitioned into a set of weights (occurrence probabilities) and updating the (partial) decision functions simultaneously as prescribed by the optimal decision function. Once this information is extracted and the appropriate decision functions updated, then the threshold is tested to decide on the presence of a targeted $R N$ as shown in the figure. If the photon is not photoelectric, then it is rejected and the next arrival is processed.

Applying statistical models of our problem, that is, each energy component is assumed independent Gaussian and the corresponding interarrival times are exponentially distributed [2] For our problem we can re-write Eq. 8 using the corresponding parameter estimates as the final sequential loglikelihood ratio radionuclide detector. That is, we require the set of targeted (true) parameters, $\left[\left\{\underline{\alpha}^{t}\right\},\left\{\underline{\epsilon}^{t}\right\},\left\{\triangle \underline{\tau}^{t}\right\}\right]$ and estimated parameters, $[\{\underline{\hat{\alpha}}\},\{\underline{\hat{\epsilon}}\},\{\triangle \underline{\hat{\tau}}\}]$. Once acquired, the decision function can be calculated and compared to the thresholds which have been estimated from ROC curve operating points.

$$
\begin{aligned}
& \Lambda\left[\Xi_{n}\right]=\Lambda\left[\Xi_{n-1}\right]+ \\
& \sum_{m=1}^{M_{\epsilon}} \sum_{\ell=0}^{L_{\epsilon}(m)} \ln \left(\frac{\left(\alpha_{m \ell}^{t}\right)^{2} \lambda_{\triangle \tau_{m \ell}}^{t}}{\sqrt{2 \pi} \sigma_{\epsilon_{m \ell}^{t}}}\right)-\ln \left(\frac{\hat{\alpha}_{m \ell}^{2} \hat{\lambda}_{\triangle \tau_{m \ell}}(n \mid n)}{\sqrt{2 \pi} \hat{\sigma}_{\epsilon_{m \ell}}(n \mid n)}\right) \\
& +\left(\hat{\alpha}_{m \ell} \hat{\lambda}_{\triangle \tau_{m \ell}}(n \mid n)-\alpha_{m \ell}^{t} \lambda_{\triangle \tau_{m \ell}}^{t}\right) \xi_{\triangle \tau}(n)
\end{aligned}
$$




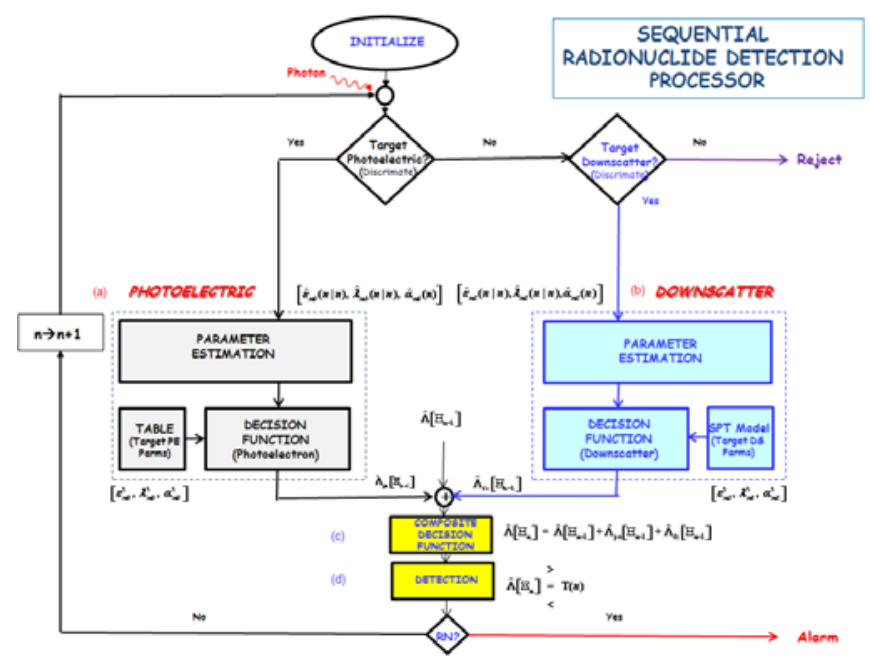

Fig. 2. Flow diagram of radionuclide detection processor: (a) Photoelectric processor. (b) Downscatter processor. (c) Decision function. (d) RN Detection

$$
+\frac{1}{2}\left(\frac{\xi_{\epsilon}(n)-\hat{\bar{\epsilon}}_{m \ell}(n \mid n)}{\hat{\sigma}_{\epsilon_{m \ell}}(n \mid n)}\right)^{2}-\frac{1}{2}\left(\frac{\xi_{\epsilon}(n)-\epsilon_{m \ell}^{t}(n)}{\sigma_{\epsilon_{m \ell}^{t}}}\right)^{2}
$$

\section{IMPLEMENTATION}

There are three phases to the implementation: (1) discrimination; (2) estimation; and (3) detection as shown in Fig. 2. The first step is to discriminate the arrival to ascertain whether or not it is one of the targeted $R N$ components, that is, either a photoelectron or a downscattered photon. Discrimination is performed using truth data from Tables [3] and the output of SPT model [7] for downscatter along with the resulting parameters using calibration data to construct confidence intervals for both energy and average interarrivals. It is here that the calibrated one dimensional SPT model is applied to obtain the targeted downscatter detection rates and occurrence probabilities for the interval constructs. Once this step is accomplished and the arrival is accepted, then the parameter estimation step is performed. Here the energy, interarrival and emission/occurrence probability are estimated using the distribution models: energy/Gaussian (linear Kalman filter), interarrival/Exponential (particle filter) and emission/occurrence probability (sequential counter). With these parameter estimates available we can then calculate the decision function (detection) by incorporating both photoelectron and downscattered arrivals. The thresholds are pre-calculated from ROC curves and the operating point (detection and false alarm probability) selected.

Sequential radionuclide detection is implemented in a channel-by-channel framework. For a given set of radionuclides, the distributions associated with each individual monoenergetic component are calculated in parallel at each channel and then combined in the detector/identifier. The sequential radiation detector has three possible choices to discern the incoming photon arrival: (1) a target photoelectron; or (2) a target downscattered photon; or (3) a background/noise photon. If it is deemed background/noise, then the photon is rejected. However, if it is a photoelectron or downscatter, then it is processed separately. Thus, two stages of discrimination are required, rather than the single stage of Refr. [8]. First, the arrival is discriminated to be a photoelectron and processed as such, if not, it is discriminated to be a downscatter photon and then processed using a model-based scheme to extract its required parameters for detection. Once extracted the appropriate (partial) decision functions are updated accordingly. Thus, the sequential radionuclide detector is implemented in a channelby-channel framework. Basically, the individual distributions are calculated in parallel at each channel and then combined in the detector/identifier. At each arrival after discrimination, the accepted channel $j k$ th photon is processed by the energy and interarrival parameter estimators $(\hat{\theta})$ providing the input to the log-likelihood ratio decision function along with the truth parameters $\left(\theta^{t} \rightarrow\left[\left\{\epsilon_{m \ell}^{t}\right\},\left\{\triangle \tau_{m \ell}^{t}\right\},\left\{\alpha_{m \ell}^{t}\right\}\right] ; \ell=\right.$ $\left.0, \cdots, L_{\epsilon}(m) ; m=1, \cdots, M_{\epsilon}\right)$ from the Tables [3] and $S P T$ model [7].

Thus, after successful discrimination the parameters are estimated and employed to calculate the log-likelihood function. These are estimated channel-by-channel ( $m \ell$-th-channel) and the overall decision function implemented sequentially (in arrival time). Once the parameters are estimated they are implemented in each channel log-likelihood partial calculation $\left(\Theta_{m \ell}(n ; \theta)\right)$ and all of the partial sums are combined along with the previous (in arrival time) log-likelihood to sequentially update the new log-likelihood at time $n$. It is then compared to the threshold to see if a detection is possible. If not, the next photon is processed and the log-likelihood updated to see if a decision can be made. This sequential radionuclide process continues until there is enough data for a decision.

\section{Table I. Sequential Radiation Detection with Scattering Discrimination}

$$
\begin{aligned}
& {\left[\epsilon_{m \ell}^{t}-\kappa_{\gamma} \sigma_{\xi} \leq \epsilon_{m \ell}(n) \leq \epsilon_{m \ell}^{t}+\kappa_{\gamma} \sigma_{\xi}\right] \quad \text { (energy) }} \\
& {\left[\triangle \tau_{m \ell}^{t}-\kappa_{\tilde{\gamma}} \sigma_{\triangle \bar{\tau}} \leq \triangle \hat{\bar{\tau}}_{m \ell}(n) \leq \triangle \tau_{m \ell}^{t}+\kappa_{\tilde{\gamma}} \sigma_{\triangle \bar{\tau}}\right] \quad \text { (interarrival) }} \\
& \underline{\text { Estimation }} \\
& \hat{\epsilon}_{m \ell}(n \mid n)=\hat{\epsilon}_{m \ell}(n \mid n-1)+K_{\epsilon_{m \ell}} i_{m \ell}(n) \quad \text { (energy) } \\
& \triangle \hat{\tau}_{m \ell}(n \mid n)=\arg \max \hat{\operatorname{Pr}}\left(\triangle \tau_{m \ell}(n) \mid \Xi_{n}\right) \quad \text { (interarrival) } \\
& \hat{\lambda}_{\triangle \tau_{m \ell}}(n \mid n)=\frac{1}{\triangle \hat{\tau}_{m \ell}(n \mid n)} \quad \text { (detection rate) } \\
& \hat{\alpha}_{m \ell}(n)=\frac{N_{\epsilon_{m \ell}}(n)}{M_{\epsilon}(n)} \quad \text { (emission probability) } \\
& \Lambda\left[\Xi_{n}\right]=\Lambda\left[\Xi_{n-1}\right]+\ln \Theta_{m \ell}\left(n ; \theta^{t}\right)-\ln \Theta_{m \ell}(n ; \hat{\theta})(\mathrm{dcn}) \\
& \underline{\text { Detection }} \\
& \begin{array}{cccc} 
& \mathcal{H}_{1} & & \\
\Lambda & \geq & \mathcal{T}_{1} & \\
& = & \text { Continue } & \text { (log-likelihood) } \\
& \leq & \mathcal{T}_{0} & \\
& \left.\Xi_{n}\right] & &
\end{array}
\end{aligned}
$$




\section{Results}

A proof-of-concept experiment was developed [8] to assess the feasibility of the sequential Bayesian processor. Three source radionuclides (cobalt $\left({ }^{60} \mathrm{Co}\right)$, cesium $\left({ }^{137} \mathrm{Cs}\right)$, barium $\left.\left({ }^{133} \mathrm{Ba}\right)\right)$ were targeted in a laboratory environment contaminated with background and extraneous sources. The sources were centered on a direct line with the HPGe detector face at a distance of $100 \mathrm{~cm}$ for $1000 \mathrm{sec}$. Each target source and background was individually counted with the results combined to generate the controlled "feasibility" data set.

The sequential Bayesian detector was applied to the "feasibility" data set. The overall results of the processing are shown in Fig. 3. We note three columns of data, the first column is the composite pulse-height spectrum (not used), with the second the composite EMS with the circles representing the discriminator output photonelectrons and the squares the downscatter photons. The final column is the decision functions for each of the targeted radionuclides. As each photon is processed, the decision function is sequentially updated until one of the thresholds (target/nontarget) is crossed (lighter crosses in figure) declaring a threat or non-threat. The results of the photoelectric and downscatter processor are shown in Fig. 3 where we observe detection times of 5.76 secs, 0.47 secs, and 0.46 secs respectively for the cobalt, cesium and barium radionuclides. This performance is expected since the "new" downscatter photon information is incorporated into each channel. The ROC operating point was $(98 \%, 2 \%)$ specifying the thresholds. Comparing these results to our previous photoelectron detector [8], we see that the results are faster for cesium $(0.47<0.68 \mathrm{sec})$ and barium $(0.46<$ $0.51 \mathrm{sec})$, but slightly slower for the cobalt $(5.76>4.05$ $\mathrm{sec})$. Few cobalt photons are available along with only a few downscatters in this realization.

The results of applying the processors to an ensemble of 100 EMS arrival are shown in Table II where we see the average detection times for each radionuclide (barium, cesium, cobalt) and each implementation (photoelectric-only (PE), photoelectric and downscatter (PE+DS). The results are reasonable for the basic and advanced (model) processors.

Thus, sequential Bayesian detector was achieved by defining a target radionuclide(s) and its monoenergetic decomposition including downscatter evolving from the underlying transport physics of the photon and measurement process. The key idea was to process the data, photon-by-photon, rejecting any extraneous non-targeted radionuclide measurements and processing only those that correspond to the targeted threat radionuclide(s) while identifying downscattered photons and extracting the underlying information for processing. The new extended processor performed quite well.

Table II. Processor Comparison: 100 EMS Ensemble Runs.

\begin{tabular}{||c|c|c|c||}
\hline \hline & ${ }^{133} \mathrm{Ba}$ & ${ }^{137} \mathrm{Cs}$ & ${ }^{60} \mathrm{Co}$ \\
\hline Processor & $\mu \pm \sigma(\mathrm{sec})$ & $\mu \pm \sigma(\mathrm{sec})$ & $\mu \pm \sigma(\mathrm{sec})$ \\
\hline PE & $0.40 \pm 0.10$ & $0.67 \pm 0.24$ & $2.68 \pm 0.49$ \\
\hline PE+DS(Model $)$ & $0.40 \pm 0.11$ & $0.53 \pm 0.22$ & $2.67 \pm 0.55$ \\
\hline
\end{tabular}

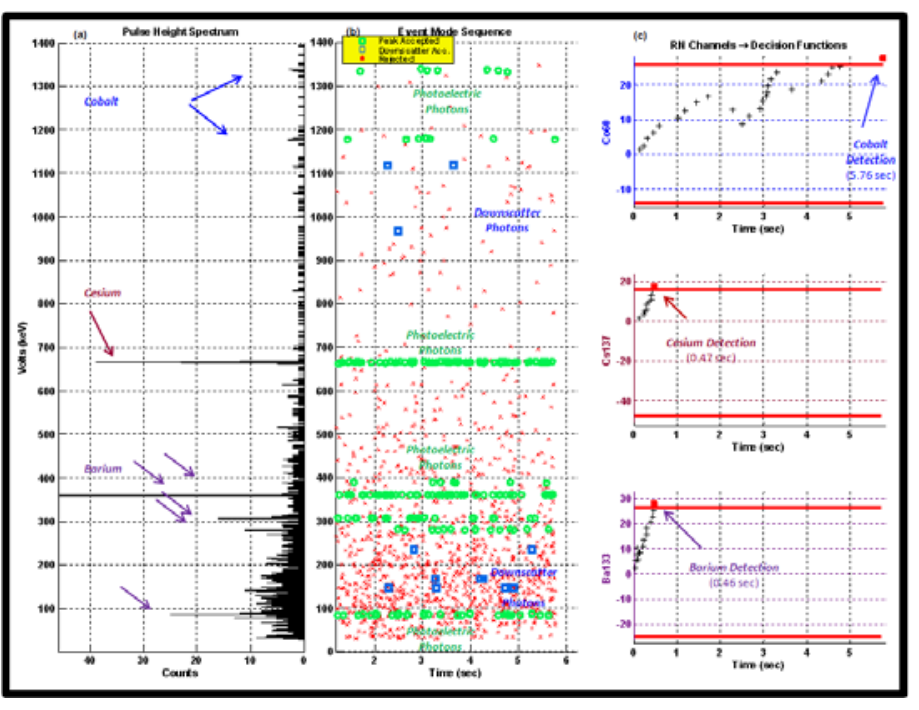

Fig. 3. Photoelectric and downscatter Bayesian detection and identification. (a) Pulse-height spectrum (after calibration). (b) EMS with discrimination (circles). (c) Log-likelihood decision functions for ${ }^{60} \mathrm{Co}$ (detection time: $5.76 \mathrm{sec}$ ), ${ }^{137} \mathrm{Cs}$ (detection time: $0.47 \mathrm{sec}$ ) and ${ }^{133} \mathrm{Ba}$ (detection time: $0.46 \mathrm{sec}$ ) radionuclide detection/identification.

\section{ACKNOWLEDGMENTS}

We would like to acknowledge the contributions of our nuclear experts, Dr. T. Gosnell, Dr. D. Slaughter, Professor S. Prussin, and Mr. D. Manatt for their encouragement, guidance and tremendous insight in helping to direct our efforts. This work performed under the auspices of the U.S. Department of Energy by Lawrence Livermore National Laboratory under Contract DE-AC52-07NA27344.

\section{REFERENCES}

[1] R. D. Evans, The Atomic Nucleus. New York, New York: McGraw-Hill, 1985.

[2] G. F. Knoll, Radiation Detection and Measurement, 3rd ed., Hoboken New Jersey: John Wiley, 2000.

[3] M. Herman, Dir., National Nuclear Data Center, Brookhaven Nat. Lab., Available: www.nndc.bnl.gov, 1986.

[4] J. V. Candy, Model-Based Signal Processing, Hoboken, New Jersey: John Wiley/IEEE Press, 2006.

[5] J. V. Candy, Bayesian Signal Processing, Classical, Modern and Particle Filtering, Hoboken New Jersey: John Wiley/IEEE Press, 2009.

[6] D. L. Snyder and M. I. Miller, Random Point Process in Time and Space. New York, New York: Springer-Verlag, 1991.

[7] D. Chambers, "A signal processing model for radiation transport," $L L N L$ Report, LLNL-TR-405952, 2008.

[8] J. V. Candy, E. Breitfeller, B. L. Guidry, D. Manatt, K. Sale, D. Chambers, M. A. Axelrod and A. Meyer, "Physics-Based Detection of Radioactive Contraband: A Sequential Bayesian Approach" IEEE Trans. Nuclr. Sci., Vol. 56, No. 6, pp.3694-3711, 2009.

[9] J. V. Candy, D. H. Chambers, E. F. Breitfeller, B. L. Guidry, K. E. Sale, M. A. Axelrod and A. M. Meyer, "Threat Detection of Radioactive Contraband Incorporating Compton Scattering Physics: A Model-Based Processing Approach," LLNL Report, LLNL-JRNL-422429, 2009.

[10] A. Papoulis and S. Pillai, Probability, Random Variables and Stochastic Processes, 4th ed., New York, New York: McGraw-Hill, 2002.

11] A. Wald, Sequential Analysis, New York, N.Y.: John Wiley, 1947 (Reprint Dover Publications, 1973). 